\title{
LA MÚSICA EN EL ÁMBITO EDUCATIVO: LAS COMUNIDADES DE APRENDIZAJE
}

\author{
ANA MARÍA GUTIÉRREZ MARTíNEZ 1 \\ Universidad de Córdoba (España)
}

\section{RESUMEN}

A lo largo de la historia, la música se ha utilizado para mejorar el bienestar físico, psíquico y emocional. En este sentido, el sistema educativo, y dentro de él, las Comunidades de Aprendizaje ${ }^{2}$, también han apostado por la música como elemento formador. Así, en el presente artículo, repasaremos el papel que la música tiene dentro del sistema de enseñanza, sus posibilidades en cada nivel educativo y su inclusión dentro de las CCAA.

Palabras clave: Música, comunidades de aprendizaje, inclusión social, atención a la diversidad, interdisciplinariedad

\section{ABSTRACT}

Throughout history, music has been used to improve the physical, mental and emotional wellbeing. In this sense, the educational system, and within it, the Learning Communities (LC), have also opted for music as an educational element. Thus, in this article, we will review the role that music plays in the education system, its possibilities in the field of secondary education and its inclusion within LC.

Key words: Music, learning communities, social inclusion, attention to diversity, interdisciplinarity

\section{Introducción}

En estos últimos años, el rápido cambio social, motivado en gran medida por los avances tecnológicos, y el frenético ritmo de vida han afectado drásticamente a la vida de las personas. Vivimos en la sociedad de la comunicación y con ello se han creado nuevas necesidades que provocan día a día un aumento en la demanda de competencias. Ahora, por ejemplo, es indispensable saber sobrellevar estados de estrés y tensión elevados y derivados de estos tiempos de constante ajetreo e incertidumbre. Por todo ello, se hace imprescindible un alto nivel de competencia personal, lo cual exige una alta cualificación en todos los niveles posibles: emocional, intelectual, profesional, social..., y no hay duda de que en ese proceso de formación, la educación juega un papel decisivo.

La sociedad del siglo XXI guarda pocas similitudes con la de épocas pasadas, por lo que los métodos educativos no pueden quedarse estancados. Si dicha metodología funcionaba en el pasado es porque se ajustaba a la sociedad de su tiempo pero, si la educación quiere avanzar a la par que la sociedad, la renovación ha de ser una máxima en ella. Así, la música puede contribuir en gran medida a desarrollar las nuevas competencias que la era tecnológica exige -creatividad, control emocional, dinamismo, empatía, habilidades sociales, etc.- y, por ello, resulta fundamental su inclusión en el currículo desde la más tierna infancia.

Del mismo modo, conviene tener presente que, si bien en el pasado la educación se basaba prácticamente en la memorización de contenidos teóricos, ahora ha de apostar por lo práctico, por el empirismo que supone vivir experiencias para aprender de ellas a todos los niveles. Así, el aprendizaje ya no queda en el aula, sino que trasciende a todos los contextos sociales donde se mueve el individuo, con carácter integrador. Bajo esta filosofía se mueven las CCAA, donde cada individuo se convierte en alumno y a la vez mentor, y donde el carácter participativo hace que el aprendizaje cobre una dimensión interactiva y práctica que permita el desarrollo y el bienestar interpersonal. En este sentido, la música es

\footnotetext{
1 Email: amagumaflauta@gmail.com
}

2 CCAA en adelante. 
una forma de comunicación universal que fácilmente puede compartirse y donde la interacción personal cobra gran importancia. No es de extrañar, por tanto, que sea una herramienta de gran utilidad en los planes educativos, dada su versatilidad y las enormes posibilidades que ofrece a nivel personal y social.

\section{Evolución de la música en el sistema educativo: desarrollo en cada nivel escolar}

La música posee gran cantidad de propiedades (Fernández-Carrión, 2011): curativas, catárticas, terapéuticas, sociales, lúdicas, hedónicas, emocionales, afectivas, formativas, éticas, espirituales, etc., lo que la ha llevado a ser un elemento fundamental en la educación de las personas. De hecho, ya en la Grecia Clásica, filósofos como Aristóteles y Platón destacaban el papel de la música en la enseñanza de los más jóvenes y de las principales clases sociales, destacando además sus múltiples virtudes éticas, morales y educativas (Grout y Palisca, 2006). Dicho pensamiento influiría profundamente en los autores medievales como Boecio, que establecía la música dentro de las disciplinas científicas del Quadrivium, y en renacentistas como Lutero, para el cual no podría concebirse la idea de un buen maestro si este no sabía cantar.

Aunque la música siempre tuvo especial relevancia en el ámbito educativo, habría que esperar a principios del siglo XX para que la enseñanza musical empezara a centrarse en principios basados en el desarrollo integral del alumnado, especialmente de aquel con necesidades especiales. Así, a lo largo de todo el siglo XX se sucedieron diversos periodos dentro de la pedagogía musical moderna, con autores de renombre que revolucionarían el panorama didáctico-musical (Sabbatela, 2004):

- La primera etapa, iniciada en 1900 y conocida como Revolución, sería encabezada por los autores de la Escuela Nueva, tales como Jacques-Dalcroze -creador de la terapia educativa rítmica- y Willems -que destaca la importancia del factor psicológico y del desarrollo de la personalidad gracias a la música-.

- La etapa de transición, que comenzó a partir de 1955, contaría con pedagogos como Ward, Kodály -haciendo hincapié en la formación vocal y auditiva a través de canciones populares, Orff -valorando el papel social y activo del individuo en el proceso musical e integrando para ello los denominados "instrumentos Orff" - y Suzuki -basando el método de aprendizaje instrumental en un proceso similar al del aprendizaje de la lengua materna.

- A partir de 1965, la etapa de Revisión estuvo representada por músicos que animaban a la potenciación de la creatividad a través de la experimentación en el aula y a la composición bajo la estética contemporánea, tales como Self, Paynter y Schafer.

- Ya en 1980, el proceso de Integración se vio enriquecido con una gran variedad de tendencias con fines dispares, como la investigación, la musicoterapia, la psicología de la música y el multiculturalismo.

- El último periodo del siglo XX, llamado ecológico y que abarca su última década, se basó en conceptos como la acústica, la ecología y la conciencia sonora. Aquí los hallazgos de su máximo representante, Schafer, supusieron un gran avance para la especialización de la educación musical y la musicoterapia.

Centrándonos en el siglo XXI, los profundos cambios sociales y la rápida evolución tecnológica hacen que las metodologías pedagógicas musicales tengan que adaptarse a los nuevos tiempos, de modo que contribuyan a formar a nivel holístico al alumnado más que a adquirir un conocimiento puramente teórico. No obstante, el diseño eficaz de la pedagogía musical parte de adaptar las posibilidades que esta ofrece a las capacidades y limitaciones del alumnado en cada etapa escolar. Así, a continuación, presentamos una clasificación de las aportaciones musicales a cada etapa escolar, de acuerdo con el desarrollo psicoevolutivo general del alumnado en las etapas de Infantil, Primaria y Secundaria (Gfeller, 2000):

- Educación Infantil. Si bien de los 0 a los 2 años el niño se guía únicamente por su experiencia sensorial, en la etapa pre-operacional -de 2 a 7 años- ya no se depende únicamente de la necesidad de experimentar los estímulos físicamente para poder entenderlos. El proceso de imitación de melodías y las ganas de cooperar y compartirlas con los compañeros va mejorando a partir de los cuatro o cinco años, pudiendo servir la música en esta etapa como una herramienta para desarrollar la empatía o la disciplina, para respetar 
los turnos en las actividades y en los juegos de grupo y para el desarrollo de otras habilidades sociales de carácter comunicativo y emocional.

- Educación Primaria. De los 7 a los 11 años -etapa de las Operaciones Concretas-, se produce un afianzamiento de los movimientos motrices, para lo cual ayuda mucho incluir el baile junto con la música. El desarrollo de la capacidad lógica lleva a los niños a ser capaces de solucionar problemas de su realidad inmediata y a procesar conceptos rítmicos y de armonía, incluso en situaciones en las que se presenten otras melodías secundarias que puedan distraer. Teniendo en cuenta esto, la música en esta etapa puede servir para afianzar y desarrollar la memoria, para incrementar el cociente intelectual-como demuestra el Efecto Mozart- o para favorecer la concentración.

- Educación Secundaria. A partir de los 11 años -periodo de las Operaciones Formales- se desarrolla la capacidad de pensar de manera abstracta, que adquiere niveles de pensamiento cada vez más complejos a medida que se vaya incrementando el número de experiencias vitales. La adolescencia supone una importante época en la que se define la identidad y, en este sentido, la música juega un papel decisivo. Muchos adolescentes se identifican con un estilo musical concreto e incluso asocian una forma de vestir, de sentir y de vivir a dicho estilo -como sucede con el heavy o el rock, por ejemplo-. Su experiencia musical llega a ser enorme si tenemos en cuenta que la música está prácticamente en todas partes. El hecho de compartir su experiencia musical y, de forma inherente, vital, con adolescentes que comparten sus mismos gustos les lleva a afianzar y a fortalecer sus vínculos sociales y su pertenencia al grupo. No obstante, también se deben potenciar la tolerancia y la curiosidad por descubrir otros estilos diferentes que les lleven a enriquecer su universo sonoro. Por todo ello, la música en esta etapa constituye un factor esencial para desarrollar valores tan importantes como la apertura de pensamiento, el enriquecimiento personal, la armonía interpersonal e intercultural, el desarrollo de las habilidades comunicativas y la expresión de emociones profundas, difíciles de expresar de otra manera.

La música, como vemos, constituye un medio perfecto para el desarrollo físico, mental y espiritual del alumnado, ya que le permite adquirir capacidades motrices básicas, le ayuda a expresarse y comunicarse de cara a la sociedad y puede llevarlo a desarrollar valores éticos que afectan al bienestar individual y social. De todo ello se ocupan las CCAA, que pretenden alcanzar el conocimiento colectivo bajo los principios de inclusión e igualdad, cosa que resulta fundamental en cuestiones como la educación especial y la atención a la diversidad ${ }^{3}$.

\section{La música dentro de las comunidades de aprendizaje}

\subsection{Concepto, principios y objetivos de las Comunidades de Aprendizaje}

Son muchos los autores que han dado una definición de CA y en todas ellas encontramos como factor común el diálogo y la participación. Así, para Ramón Flecha García (2002, p. 11) una CA supone un proyecto que "apuesta por el aprendizaje dialógico mediante los grupos interactivos, donde el diálogo igualitario se convierte en un esfuerzo común para lograr la igualdad educativa (...)". Para Valls (2000, p. 8):

Comunidad de Aprendizaje es un proyecto de transformación social y cultural de un centro educativo y de un entorno para conseguir una sociedad de la información para todas las personas, basada en el aprendizaje dialógico, mediante una educación participativa de la comunidad, que se concrete en todos sus espacios, incluida el aula.

Respecto al aprendizaje dialógico mencionado anteriormente, este se entiende como un tipo de aprendizaje que no se reduce al aula en forma de interacción profesor-alumno alumno-profesor, sino que incluye a todas las personas que rodean al escolar, influyendo con ello en su proceso de aprendizaje (Aubert, García y Racionero, 2009). Así, esta nueva concepción se aleja del modelo educativo tradicional, pues ahora la educación ya no se limita al centro de enseñanza, sino que permanece en todos los

3 Ver epígrafe 3.2. 
aspectos de la vida del individuo, extrapolándose a contextos escolares y extraescolares. De esta manera, la educación proviene de cada uno de los individuos que interactúan con el sujeto, convirtiendo su proceso vital en un contexto educativo e implicando a todos los sectores sociales que lo rodean: comunidad educativa, familia, amigos y sociedad en general. Según este principio, todos los sujetos actúan a su vez como modelos y referentes, favoreciendo la apertura mental, la tolerancia, el enriquecimiento y la formación a través de "iguales". Todas estas circunstancias favorecen el concepto de comunidad y la idea de que, al formar parte de ella, el bien individual contribuirá al bien grupal y viceversa.

Para Coll (2001), este nuevo espacio interactivo abarca cuatro grandes categorías: el aula Classroom-based Learning Communities-, el centro educativo -School-based Learning Communities-, la ciudad, comarca o región de residencia de la persona -Community-based Learning Communities, Community Learning Networks, Learning Cities, Learning Towns, Learning Regions- y el entorno virtual Virtual Learning Communities-. Resultado de la interacción de todas ellas es la creación de un conocimiento colectivo (Reigeluth, 2013) basado en procesos de aprendizaje, trabajo colaborativo y consenso social.

Por otra parte, Bielaczyc y Collins establecen catorce principios para el diseño de CCAA eficaces en el aula (Reigeluth, 2013):

- El principio del crecimiento de la comunidad, basado en incrementar el conocimiento y las habilidades del grupo. A esto contribuyen metodologías como la técnica puzzle de Aronson.

- El principio de objetivos emergentes, centrado en construir dicho conocimiento a partir de las demandas y necesidades del aula en el proceso de enseñanza-aprendizaje.

- El principio de articulación de objetivos pretende establecer acuerdos en el aula y favorecer el sentimiento de grupo, fijando los objetivos y los criterios de evaluación a partir de un consenso.

- El principio de metacognición destaca el papel de la reflexión sobre el propio aprendizaje, modificando lo necesario para optimizar el rendimiento.

- El principio de superación de los límites es fundamental para conseguir un aprendizaje significativo y desarrollar las potencialidades de cada individuo y del grupo.

- El principio del respeto resalta la importancia de respetar, comprender y valorar las aportaciones de cada miembro del grupo.

- El principio de aceptación del fracaso insiste en convertir las experiencias negativas en un aliciente para crecer, impulsando el concepto de resiliencia.

- El principio de dependencia estructural implica que las actividades realizadas a nivel individual repercuten directamente en el trabajo del grupo, lo que favorece la simbiosis entre sus miembros.

- El principio de preeminencia de la profundidad sobre la amplitud destaca la necesidad de respetar los tiempos en el proceso de maduración de conceptos, algo fundamental en el desarrollo del potencial humano.

- El principio de la diversidad parte del aprendizaje entre iguales en el cada estudiante enseña a sus compañeros los conocimientos de las áreas en las que se sienten más preparados y capaces, favoreciendo que todos sean maestros de todos.

- El principio de la multiplicidad de vías de participación implica que las actividades propuestas sean diversas y estén al alcance de todos para alcanzar el éxito.

- El principio de mecanismos para compartir vuelve a remarcar la necesidad del aprendizaje entre iguales.

- El principio de negociación parte del consenso entre los miembros del grupo para la realización de cualquier actividad.

- El principio de calidad de los productos destaca la necesidad de compartir el conocimiento creado por el grupo con otras personas ajenas a él, teniendo muy en cuenta la valoración externa.

En cuanto a los objetivos de las CCAA, el principal de todos es entender y crear conocimiento como un bien colectivo, de manera que la sociedad sea construida por todos y para todos, posibilitando que 
cada individuo se convierta a su vez en alumno y mentor. Sin embargo, esto implica retos como la creación de un sistema de enseñanza mucho más flexible y abierto al cambio, capaz de renovarse y de actualizarse día a día, con el objetivo de evolucionar con y para la sociedad. En este sentido, Paolo Freire (2003) propone la transformación de las personas y del mundo a través de la educación, por lo que se hace necesario abandonar la idea tradicional de educación "bancaria" -en la que el profesor imparte contenidos y el alumno los almacena para plasmarlos en un examen- por un nuevo modelo en el que los alumnos participan de forma activa en su propia comunidad, asumiendo las responsabilidades que ello implica (García, 2002, p. 2). Otros objetivos que se persiguen son:

- Conseguir un sistema educativo más igualitario en el que las diferencias de sexo, religión, raza, poder adquisitivo, capacidades, etc. no supongan factores de exclusión.

- Unificar el conocimiento como una gran construcción colectiva en la que cada disciplina de saber interactúa con el resto y no de manera aislada, conformando un todo y favoreciendo la interdisciplinariedad.

- Valorar el proceso de aprendizaje por encima del resultado final, entendiendo cada contexto de aprendizaje como un fin en sí y no como un medio para alcanzar un conocimiento superior.

- Ampliar la idea de "conocimiento" por encima de la simple adquisición de saberes, pues también se desarrollan habilidades y capacidades que constituyen un todo.

- Integrar a grupos diversos dentro de un solo colectivo, el de la sociedad, favoreciendo la tolerancia, el respeto y la apertura a lo nuevo.

- Abandonar el esquema de educación y sociedad basado en la jerarquía y el poder, implicando a todos los colectivos como agentes activos de conocimiento.

- Favorecer el trabajo en grupo, el diálogo, el consenso, la adquisición de responsabilidades y el desarrollo del máximo potencial humano.

En definitiva, los objetivos perseguidos son todos aquellos que permitan crear una sociedad en la que el concepto de colectividad y el sentimiento de comunidad superen las limitaciones que actualmente supone la mentalidad individualista imperante en la sociedad actual y en la que se dejan de lado valores tan importantes como la solidaridad o el amor al prójimo.

\subsection{Inclusión social y atención a la diversidad}

Las CCAA constituyen un espacio perfecto para la integración y la inclusión social, ya que en ellas prima el consenso y el diálogo para llegar a construir significados comunes, al margen de ideologías individuales. Esto permite que la diferencia se conciba como una posibilidad de enriquecimiento del conocimiento inicial y no como un factor de exclusión. El resultado de ese intercambio recíproco de saberes y aprendizajes supone una revalorización del conocimiento individual en favor del conocimiento colectivo, lo que favorece la autoestima, la integración y el sentimiento de grupo.

En cuanto a la atención a la diversidad, Flecha y Puigvert (2002, p. 18) destacan que "las comunidades de aprendizaje no quieren adaptarse a la diversidad (es decir, a la desigualdad) sino transformar la escuela y su contexto social hacia la utopía de convertirla en un proyecto educativo igualitario". Para no llevar a equívocos, es necesario aclarar esta afirmación, ya que detrás de ella se esconde la verdadera filosofía de una CA. Para una CA, el hecho de que en la normativa referente a educación exista un apartado dedicado a la atención a la diversidad ya supone un factor de exclusión, pues da a entender que este colectivo se merece un trato diferente al que recibiría cualquier miembro "no diverso" (Flecha y Puigvert, 2002). No obstante, se trata de un aspecto un tanto delicado y abierto a interpretaciones.

Para entender mejor la delicadeza del término "atención a la diversidad" dentro de una CA pondremos un ejemplo que aclarará las posibles dudas. Llevando la CA al ámbito educativo, imaginemos que en el aula hay un niño ciego, otro con problemas psicomotrices y otro con problemas mentales, por ejemplo. Aunque estos alumnos también aporten conocimientos al saber colectivo que se crearía entre todo el aula, también es cierto que necesitarían una adaptación de algunos de los métodos utilizados para que 
ellos puedan nutrirse de ese conocimiento colectivo. Por ejemplo, el niño ciego no podría percibir el contenido visual, por lo que habría que transmitirlo de alguna manera por vía auditiva o por cualquier otro medio por el que pudiera percibirlo. En el caso del niño con problemas psicomotrices, su coordinación y movimientos no serían tan ágiles como los del resto de compañeros, por lo que se podrían plantear otros métodos en las actividades físicas -si las hubiera-. En cuanto al niño con problemas mentales es posible que hiciera falta adaptar los contenidos a su capacidad y hubiera que buscar métodos de estimulación individualizados. En este sentido, se aplica el término "atención a la diversidad" para hacer mención a los métodos individualizados necesarios para que alumnos de este tipo asimilen los contenidos, sin infravalorar sus capacidades ni su potencial.

\subsection{Interdisciplinariedad e interactividad}

Si el conocimiento creado en una CA parte de la interrelación de saberes de los miembros del grupo, es normal que se produzca una fusión de conocimientos referentes a diferentes campos de conocimiento. Si bien cada profesor es experto en su materia, las CCAA pretenden que el personal docente adquiera el compromiso de interactuar y aunar esfuerzos para crear un conocimiento colectivo holístico e integral. Esta es, entre otras, la base del aprendizaje significativo que permite al alumnado poner en práctica en su vida diaria todo lo aprendido en el aula (Rusinek, 2004). Los conocimientos, habilidades, capacidades y actitudes aprendidas en clase son producto de todo un proceso de convivencia y socialización dentro del centro educativo. Por ello, no tiene sentido que cada profesor desempeñe su labor de una forma aislada, sino que cada parcela ha de tener su lugar propio fusionándose a su vez con los demás, aspecto al que la música contribuye en gran medida, pues puede incluirse en prácticamente todas las asignaturas: matemáticas -ejemplificado en conceptos como el ritmo y la armonía-, lengua -el canto mejora la vocalización y el lenguaje-, educación física -la danza unida a la música mejora la coordinación y la psicomotricidad-, lengua extranjera -con el canto de melodías en otro idioma-, informática -con la manipulación de programas de creación y edición musical-, física -con el análisis de aspectos acústicos, plástica -"dibujando la música" para potenciar la creatividad y la expresión de sentimientos-, ciencias naturales -al tratar el tema de la contaminación acústica o del reciclaje mediante la construcción de instrumentos con materiales de desecho-, etc.

Por otra parte, el factor interactivo es fundamental en una CA, ya que no solo está formada por los alumnos y profesores que conviven en el aula, sino que implica a todos los individuos que rodean al sujeto y participan de alguna manera en su vida. En este sentido, el pedagogo Paolo Freire (2003) destaca la necesidad de basar el proceso formativo en un diálogo que incluya a toda la comunidad y que permita la planificación conjunta del aprendizaje.

Partiendo de esta idea, podemos distinguir varios grupos de miembros implicados de forma directa en el desarrollo de este sistema de aprendizaje. Así, como principal agente, encontramos al alumnado en sí, que es sobre el que se centra principalmente el proceso de aprendizaje -aunque hay que tener presente que en una $\mathrm{CA}$ todos sus miembros son de alguna manera alumnos, porque constantemente están aprendiendo unos de otros mediante el aprendizaje dialógico de iguales-. Este factor favorece la inclusión de todos los alumnos, sin distinción, haciendo que la diferencia y el contraste de opiniones sea un factor de enriquecimiento y no de rechazo o exclusión.

Por otra parte, dentro del ámbito escolar, el profesorado ejerce un importante papel en el proceso de aprendizaje, cumpliendo una función de guía y sirviendo de referente o modelo en el proceso de construcción de la identidad. Para ello, ha de evolucionar en su papel y no vertebrar su enseñanza bajo el patrón de jerarquía de los modelos tradicionales, en los que se establece una relación de dependencia y autoridad (Fernández-Carrión, 2011). Ahora el docente ha de ser capaz de empatizar con el alumno, convirtiéndose en un apoyo constante sobre el que poder expresar con naturalidad sus inquietudes, estableciéndose con ello una relación de complicidad y simbiosis. La comprensión y el apoyo percibidos mejoran mucho el proceso de aprendizaje y por eso es fundamental tratar aspectos personales que puedan llevarle a mejorar su proceso de enseñanza, adaptándolo a sus necesidades. Estaríamos hablando de un modelo de enseñanza individualizado en el que el profesor podría entenderse más como un tutor personal y un negociador. Aunque esta situación pudiera parecer utópica -ya que el docente tiene numerosos alumnos y resultaría casi imposible poder profundizar en la situación de cada uno de 
ellos- la creación de grupos más reducidos o la posibilidad de compartir o complementar tal labor con otros miembros de la comunidad educativa podría facilitar mucho el proceso.

En cuanto al resto de miembros que forman parte de la comunidad educativa, estos forman parte del proceso de convivencia del alumno, tanto dentro como fuera del centro. No podemos pensar que esas personas actúen de manera casual y aislada, pues también suponen una importante fuente de influencia, por lo que también han de procurar participar en ese conocimiento colectivo desde un punto de vista ético y sinérgico. Ejemplo de esta clase de miembros serían, dentro del centro educativo, los conserjes, profesores que no le dan clase al alumno en cuestión, compañeros de otras clases, etc.

Por otra parte, fuera del ambiente escolar, también existen otros factores de influencia $y$, de entre todos, la familia ejerce un papel decisivo. Flecha García y Puigvert (2002, p. 20) apuntan que "el aprendizaje depende cada vez menos de lo que ocurre en el aula y cada vez más de la correlación entre lo que ocurre en las aulas, en la calle o en la cocina". Esto supone un cambio de paradigma respecto a la concepción del proceso de aprendizaje, pues ahora los conocimientos adquiridos pueden extrapolarse a cualquier ámbito de la vida y no solo al escolar. En este sentido, si tenemos en cuenta que la familia es la principal fuente de educación desde el momento del nacimiento y que suele ser el agente con el que el individuo interactúa más, no podemos dudar de la importancia que va a tener en el proceso de enseñanza y aprendizaje, superando el papel del docente o de la comunidad educativa en su conjunto. Es por esta razón por la que las CCAA destacan la necesidad de formar a las familias para que sepan transmitir valores y actitudes de cara a la vida en sociedad.

Finalmente, el último -aunque no por ello menos importante- agente que conforma una CA es la sociedad en conjunto, ya que el ser humano es un ser sociable por naturaleza y, por eso, ha de aprender a convivir en ella y con ella. Hasta ahora, las relaciones sociales se basaban en gran medida en el poder que el grupo dominante ejercía sobre el grupo minoritario, contribuyendo así a la segregación y a la exclusión. Las CCAA apuestan por lo contrario, pretendiendo crear una sociedad más justa e igualitaria en la que los principios de solidaridad, respeto y ayuda mutua sean los motores de un nuevo modelo social basado en el sentimiento de colectividad. En consecuencia, se favorecerá la creación de intereses comunes, rompiendo con las limitaciones del materialismo y del individualismo, tan propias del siglo XXI. De esta forma, la sociedad y los valores que establece determinan en gran medida la clase de personas en la que se convierten sus miembros. Es tarea de todos caminar hacia unos nuevos valores más respetuosos con nuestros semejantes y que favorezcan la unidad y la cooperación.

\subsection{Proyectos de Comunidades de Aprendizaje}

Las CCAA son una realidad creciente que se están desarrollando en la actualidad en diferentes países, con unos resultados tan eficaces y positivos que se están extendiendo a nivel internacional (Fernández-Carrión, 2011). Como ejemplo de ellas, mostramos dos modelos -FESNOJIV y Proyecto LOVA- que se centran principalmente en la inclusión del colectivo al que van dirigidos, de manera que se favorezca un panorama igualitario e integrador.

\section{-FESNOJIV}

La Fundación del Estado para el Sistema Nacional de las Orquestas Juveniles e Infantiles de Venezuela (FESNOJIV) -también denominada "El Sistema"- es una propuesta fundada por José Antonio Abréu donde la música actúa como medio de inclusión social dentro de colectivos que sufren un nivel de pobreza extremo. Así, bajo el pretexto de la formación de una orquesta y su correspondiente desarrollo, se crea un espacio comunitario donde el aprendizaje musical y, sobre todo, personal y social, es el principal motor. Aquí se trata de fomentar el amor por la música como forma de motivación y de futuro profesional entre personas que, debido a sus escasos recursos económicos y su alto riesgo de exclusión social, se encuentran en una situación constante de desesperanza.

Bien es sabido que estudiar música no está al alcance de cualquiera, pues los gastos que conlleva en ocasiones ascienden a cifras muy elevadas. Por ello, "El Sistema" proporciona a los niños del programa todos los recursos necesarios para llevar a cabo su desarrollo musical. Para las familias, esto supone un 
halo de esperanza al poder ver aspiraciones hacia un futuro mejor y una forma de sentirse escuchados, ya que la FESNOJIV apoya e implica a todo el colectivo familiar, aportando todo lo necesario para que sus músicos no dejen la orquesta debido a factores económicos. Con este proyecto, se ofrece a los niños una oportunidad única para aprender música y enriquecerse con ella, evolucionando a su vez como personas y favoreciendo la inclusión social al evitar que estos jóvenes se muevan en ambientes delictivos.

Aquí, la metodología utilizada trata de educar en unos casos y de reeducar en otros a personas que carecen de recursos y que han de desenvolverse en ambientes hostiles cargados de dificultades y con escasas posibilidades laborales. Es por ello que la música no solo se concibe como una práctica orquestal o como una formación puramente academicista, sino que aprovecha todo lo que una orquesta demanda para que sus miembros aprendan a convivir, a desarrollar técnicas de trabajo colaborativo y en equipo, a asumir responsabilidades, a adquirir compromisos, a entender que el trabajo individual afecta al desarrollo del grupo y viceversa y, en definitiva, a desarrollar al máximo sus capacidades y potencialidades musicales y personales.

Del mismo modo, para que el éxito esté garantizado, el trabajo se adapta al desarrollo psico-evolutivo propio de cada edad, de manera que los niveles iniciales están centrados en la expresión corporal y, a medida que se asciende, se pasa al trabajo del canto, del ritmo y de la interpretación, primero de la flauta dulce y de la percusión y, finalmente, de un instrumento propio.

Los resultados de dicho proyecto han sido muy favorables, como demuestra el caso de Gustavo Dudamel que, gracias a la educación recibida desde los cuatro años en "El Sistema", se ha convertido en uno de los principales directores de orquesta del mundo. La idea de crear orquestas para jóvenes en riesgo de exclusión social se ha extendido a otros países y ya se ha puesto en marcha en 25 de ellos, como Argentina, Austria, Canadá, Ecuador, Estados Unidos, India o Inglaterra.

\section{-Proyecto LOVA}

Desarrollado por Mary Ruth McGinn y Ellen Levine en 2001 con el nombre de "Creating Original Opera", este proyecto se basa en la creación de una Ópera como Vehículo de Aprendizaje. El programa parte del Metropolitan Opera Guil de Nueva York y hace uso de métodos de enseñanza innovadores y alternativos pensados para desarrollar el máximo potencial posible.

En la misma línea que "El Sistema", el Proyecto LOVA trata de crear una comunidad de aprendizaje en la que los alumnos desarrollen su propia obra a partir del trabajo en grupo, compartiendo inquietudes y colaborando por un mismo objetivo. Así, cada grupo de alumnos asume una responsabilidad y un "oficio" propio, relacionado con el mundo operístico. De esa manera, cada grupo se encarga de una tarea concreta -composición de la música, creación del decorado, redacción del libreto, realización del vestuario, interpretación musical, etc. - teniendo en cuenta la labor del resto de grupos y caminando en la misma dirección. Se trata pues de un ambicioso proyecto con carácter interdisciplinar que contribuye con creces al desarrollo personal y social del alumnado, incrementando su autoestima y su desarrollo emocional, intelectual y profesional. Aquí, los alumnos toman la iniciativa y se convierten en creadores y artistas con espíritu crítico y capacidad de diálogo, creando un producto común y en equipo donde lo importante, más que el resultado final, es el proceso para llegar a él.

La organización que se utiliza afecta tanto al alumnado como al profesor, dando lugar a nuevos roles basados más en la empatía y la guía profesional que en la simple transmisión de conocimientos. En consecuencia, el alumno ya no depende del profesor en su proceso de aprendizaje, sino que se constituye como responsable directo de su aprendizaje y toma de decisiones. El profesor actúa como un "consejero" que orienta e interviene si fuera necesario para completar el trabajo del alumno a partir de su propia experiencia. Se establece así una alta simbiosis basada en el aprendizaje mutuo entre ambas partes y extrapolada a su vez al ámbito familiar, ya que las familias también orientan al alumnado en su labor, transmitiendo sus conocimientos en las parcelas que más dominen y contribuyendo al desarrollo de la autonomía personal. Por ejemplo, en la labor de vestuario, las familias pueden guiar a sus hijos para elegir las telas adecuadas, diseñar los trajes y elaborarlos. La interacción social es constante y esto se traduce en una mejora del desarrollo cognitivo, social y emocional. 
En definitiva, la metodología seguida consiste en convertir el aula en una compañía operística y emplear el año académico en crear la obra, impartiéndose las diferentes asignaturas y desarrollando las competencias de cada una de ellas bajo un mismo hilo conductor: la ópera.

\section{Conclusiones}

Las CCAA han sido llevadas a la práctica en diversos contextos y han demostrado teorías sobre la mejora de la convivencia y la superación del fracaso escolar, siendo avaladas por la comunidad científica internacional (Díez y Flecha, 2010).

Por otra parte, la música es una disciplina que ofrece grandes posibilidades y beneficios en todos los niveles de la persona -físico, mental, intelectual, anímico, social, espiritual...-. El fenómeno sonoro está presente prácticamente en todos los contextos que rodean al individuo y, por eso, resulta muy fácil acceder a él.

Combinado las ventajas de las CCAA y de la música como herramienta de bienestar personal y grupal, pueden diseñarse nuevas metodologías y modelos educativos eficaces, adaptados a las verdaderas necesidades de la sociedad del siglo XXI. Descubrir a través de la música valores que buscan la mejora del grupo y llevarlos al resto de la sociedad permite transformar el mundo desde una perspectiva más adaptativa y ecológica.

Por todo ello, y aunque ya se han diseñado en España algunos proyectos similares a los mencionados en este artículo, se propone el diseño futuro de nuevas CCAA que utilicen la música como hilo conductor dentro del aula, fomentando desde edades tempranas la creatividad y la solución de problemas grupales bajo la máxima de que el verdadero beneficio no es solo el individual, sino también el grupal. Si bien es cierto que se está avanzando en la dirección correcta, aún queda mucho por hacer y para ello es fundamental que la música sea revalorizada tanto a nivel artístico como a nivel de herramienta de bienestar social.

\section{Referencias bibliográficas}

Aubert, A., García, C. y Racionero, S. (2009). El aprendizaje dialógico. Revista Cultura y Educación, 21(2), pp. 129-139.

Coll, C. (2001). Las Comunidades de Aprendizaje y el futuro de la educación: el punto de vista del Fórum Universal de las Culturas. Simposio Internacional sobre las Comunidades de Aprendizaje, Barcelona, 5-6 de octubre de 2001, pp. 1-17.

Davis, W.B., Gfeller, K.E. y Thaut, M.H. (2000). Introducción a la musicoterapia: teoría y práctica, 3. Barcelona: Boileau Editorial de Música.

Díez, J. \& Flecha, R. (2010). Revista Interuniversitaria de Formación del Profesorado, 67(24-1),19-30.

Fernández-Carrión, M. (2011). Proyectos Musicales Inclusivos. Revista Tendencias Pedagógicas, 17, 74-82.

FESNOJIV. Fundación del Estado para el Sistema Nacional de las Orquestas Juveniles e Infantiles de Venezuela. Recuperado de http://www.fesnojiv.gob.ve/

Flecha, R. y Puigvert, L. (2002). Las comunidades de aprendizaje: una apuesta por la igualdad educativa. REXE: Revista de estudios y experiencias en educación, 1(1), 11-20.

Freire, P. (2003). Pedagogía del oprimido. Madrid: Siglo XXI.

García, N. (2002). Las comunidades de aprendizaje. Sistemas de trabajo con las TICs en el sistema educativo y en la formación de profesionales. Revista de Educación a Distancia, 6, 1-10.

Grout, D.J. y Palisca, C.V. (2006). Historia de la música occidental 1 (Edición Ampliada). Madrid: Alianza Editorial.

Proyecto LOVA. Recuperado de http://www.proyectolova.es 
Reigeluth, Ch.M. (2013). Instructional-design theories and models: A new paradigm of instructional theory, Vol. II, pp. 269-292. London: Routledge, Taylor and Francis Group.

Rusinek, G. (2004). Aprendizaje musical significativo. Revista Electrónica Complutense de Investigación en Educación Musical, 1(5), 1-16. Recuperado de http://pendientedemigracion.ucm.es/info/reciem/.

Sabbatella, P. (2004). Intervención musical en el alumnado con necesidades educativas especiales: delimitaciones conceptuales desde la pedagogía musical y la musicoterapia. Revista de Ciencias de la Educación, 20, 123-140.

Valls, R. (2000). Comunidades de aprendizaje: una práctica educativa de aprendizaje dialógico para la sociedad de la información. Universidad de Barcelona, Tesis Doctoral.

Fecha de recepción / Received: 05/03/2016

Fecha de aceptación / Accepted: 21/05/2016 